nephron

Practice
Nephron 2018;140:111-115

DOI: 10.1159/000493203
Received: June 14, 2018

Accepted after revision: August 10, 2018 Published online: September 11, 2018

\title{
Endpoints for Clinical Trials of Acute Kidney Injury
}

\author{
Paul M. Palevsky \\ Renal Section, Medical Service, VA Pittsburgh Healthcare System, and Renal-Electrolyte Division, Department of \\ Medicine, University of Pittsburgh School of Medicine, Pittsburgh, PA, USA
}

\section{Keywords}

Acute kidney injury $\cdot$ Clinical trial $\cdot$ Creatinine $\cdot$ Endpoints

\begin{abstract}
Background: The selection of appropriate endpoints is critical to the success of any clinical trial. Endpoints need to be precisely defined and need to provide an appropriate balance between sensitivity for detection of the effects of the intervention of interest and specificity for the outcome of interest. Summary: In acute kidney injury (AKI), the 2 most common endpoints utilized either rely on small, short-term changes in serum creatinine or are a composite of longer term major adverse kidney events, including death, need for dialysis, and a persistent decline in kidney function. Key messages: Numerous issues affecting the potential utility of these endpoints are discussed that need to be carefully considered when selecting appropriate endpoints in the design of AKI trials.

(c) 2018 S. Karger AG, Basel
\end{abstract}

The selection of appropriate endpoints is critical to the success of any clinical trial. Precisely defined trial endpoints need to provide an appropriate balance between sensitivity for detection of the effects of the intervention and specificity for the outcome of interest. This has been particularly challenging in trials of acute kidney injury (AKI) for which a wide variety of potential endpoints have been proposed or utilized. These have ranged from the short-term changes in serum creatinine and or urine output that comprise the consensus definitions of AKI [1-3], to longer term outcomes, reflecting the risks of AKI on development or progression of chronic kidney disease and mortality [4]. In the following perspective, the limitations of several of the more commonly employed endpoints are considered.

\section{Short-Term Increases in Serum Creatinine}

In the simplest conceptual model for trials evaluating therapies for the prevention or treatment of early AKI, a clinical insult such as sepsis, hypotension, cardiac surgery, or nephrotoxin exposure is responsible for causing

Contribution from the AKI and CRRT 2018 Symposium at the 23rd International Conference on Advances in Critical Care Nephrology, Manchester Grand Hyatt, San Diego, CA, USA, February 26 - March 1, 2018. This symposium was supported in part by the NIDDK funded University of Alabama at Birmingham-University of California San Diego O'Brien Center for Acute Kidney Injury Research (P30DK079337).

\section{KARGER}

๑) 2018 S. Karger AG, Basel

E-Mail karger@karger.com

www.karger.com/nef
Dr. Paul M. Palevsky

VA Pittsburgh Healthcare System

University Drive, Room 7E123 (111F-U)

Pittsburgh, PA 15240 (USA)

E-Mail palevsky@pitt.edu 


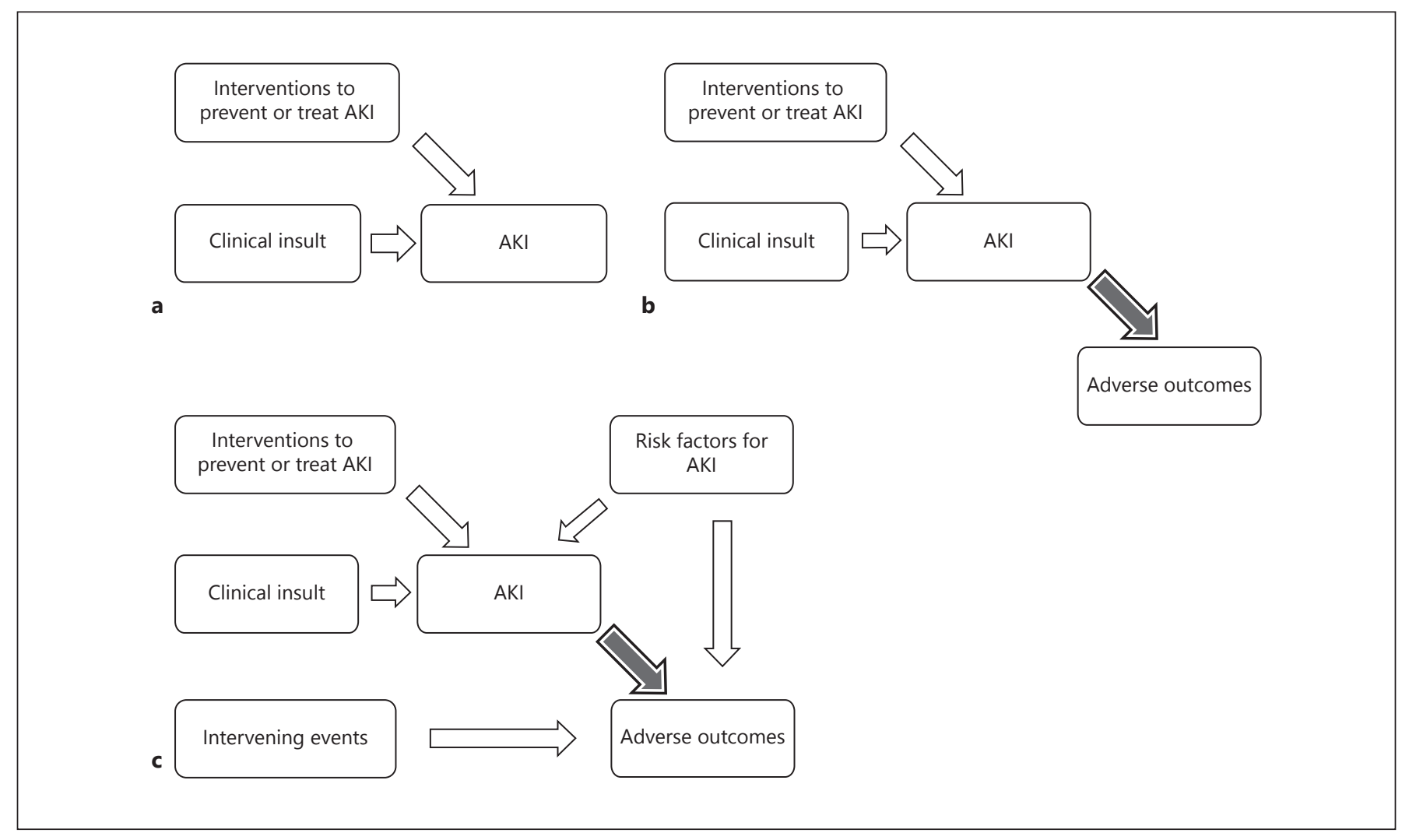

Fig. 1. Conceptual models for endpoint selection in clinical trials of AKI. a A clinical insult contributes to the development of AKI and an intervention is provided to prevent or decrease the progression of AKI. The clinical endpoint is the short-term change in kidney function, assessed by change in serum creatinine. b AKI development of AKI is associated with subsequent adverse outcomes, such as death, need for dialysis, or persistent decline in kidney

kidney injury and an intervention is provided to prevent or ameliorate that injury (Fig. 1a). Short-term increases in serum creatinine, as are used to define AKI are therefore an obvious endpoint that can, and have been, employed in clinical trials. Prior to the development of the RIFLE criteria, the first consensus definition of AKI, a multitude of definitions of AKI were employed as endpoints [5]. The RIFLE criteria [1], and the subsequent AKIN [2] and KDIGO [3] revisions provided standardization, establishing small absolute and relative increments in serum creatinine as the definition of AKI. However, in the original construct of the RIFLE criteria, the increments in serum creatinine corresponding to the categories of Risk ( $50 \%$ increase in serum creatinine), Injury (100\% increase in serum creatinine), and Failure $(200 \%$ increase in serum creatinine) were proposed as graded definitions, with decreasing sensitivity and increasing function (open arrow). The intervention to prevent or decrease the progression of AKI decreases the development of these outcomes (filled arrow). c Risk factors for the development of AKI are also risk factors for the development of the subsequent adverse outcomes. Intervening events between the intervention and endpoint ascertainment may also contribute to the development of these events. AKI, acute kidney injury.

specificity for kidney injury rather than as stages of severity of injury. The use of a definition with high sensitivity augments the expected event rate, potentially permitting a smaller sample size in clinical trials, but with the risk that "true" AKI events will be masked by false-positive events.

There are multiple reasons for the lack of specificity associated with small changes in serum creatinine. First, while these changes define fluctuation in kidney function, they are not specific for the etiology. AKI is a heterogenous condition. While the goal in a trial is generally to prevent or mitigate intrinsic injury, many events detected by small changes in serum creatinine will primarily be functional, driven by hemodynamic factors not associated with parenchymal injury. For example, while blockade of the renin-angiotensin system (RAS) is associated with increased AKI following cardiac sur- 


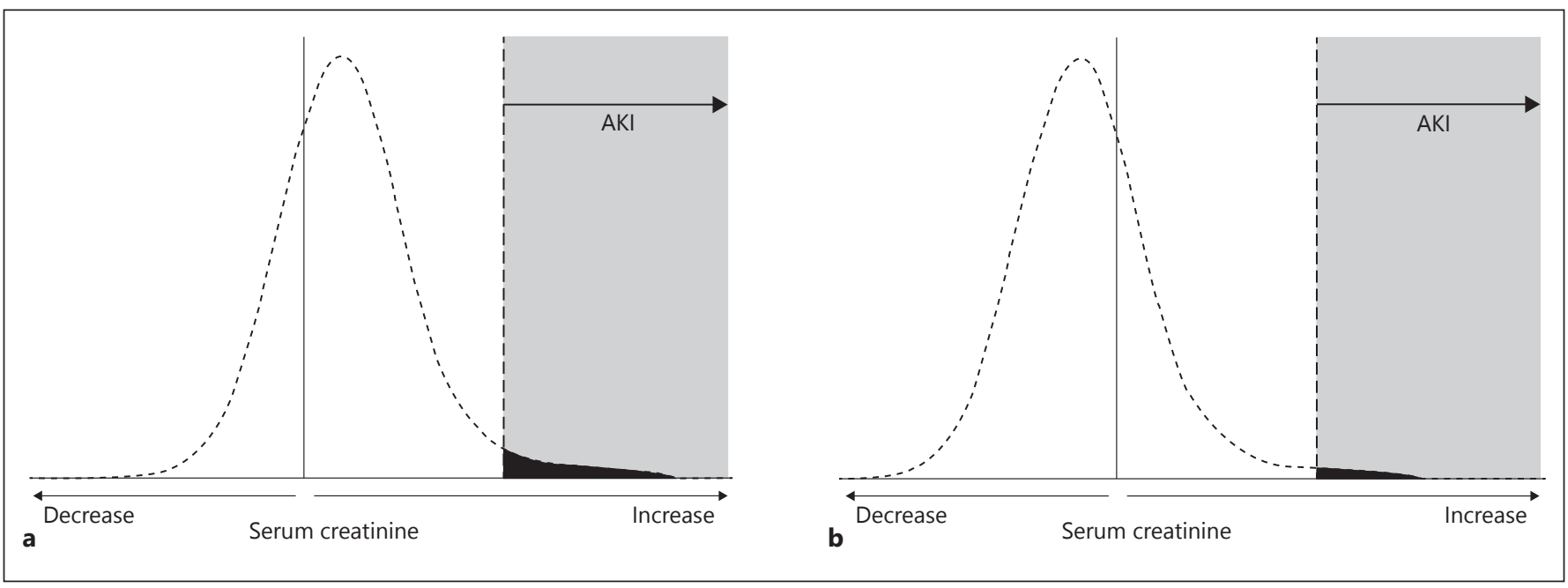

Fig. 2. Effect of small changes in serum creatinine on apparent incidence of AKI. a Distribution pattern of change in serum creatinine after an exposure associated with the development of AKI. The vertical line represents no change in serum creatinine. The curve represents the distribution in change in serum creatinine, with an overall increase in serum creatinine concentration. Increases that are greater than a defined value (shaded area) are clas-

gery, there is a dissociation between these AKI events and increases in markers of tubular injury [6]. Second, worsening of kidney function must be considered in context. Although AKI is generally associated with increased mortality, decrements in kidney function associated with RAS blockade in patients with heart failure can be associated with decreased rather than increased mortality [7]. Third, although creatinine variability is associated with increased mortality risk [8], this association is insufficient to define creatinine variability as equivalent to AKI. The combination of intra-patient biological variation and intrinsic analytic variability in serum creatinine is not insignificant, with greater variation in patients with underlying $\operatorname{CKD}[9,10]$. In one analysis, creatinine variability resulted in a false-positive rate of AKI exceeding $30 \%$ among patients with a serum creatinine $\geq 1.5 \mathrm{mg} / \mathrm{dL}$ when the AKIN and KDIGO definitions were employed [10]. Finally, interventions may alter serum creatinine concentration independent of any nephroprotective effect (Fig. 2). A lowering in serum creatinine resulting in a leftward shift in the distribution of the change in serum creatinine following a potentially nephrotoxic exposure may decrease the apparent incidence of AKI in the absence of a true decrease in toxicity. An extreme example of this is the effect of prophylactic continuous renal replace- sified as AKI. b The distribution pattern of change in serum creatinine with an intervention that decreases serum creatinine independent of a nephroprotective effect. The distribution curve is shifted to the left, as compared to (a), resulting is classification of a smaller number of individuals as having developed AKI, despite the absence of a true nephroprotective effect. AKI, acute kidney injury.

ment therapy (RRT), on prevention of AKI following angiography [11]. Continuous therapy directly lowers the serum creatinine concentration, invalidating the use of change in serum creatinine as compared to baseline as an endpoint for demonstration of nephroprotection. Similar, although less extreme confounding may be seen with multiple other interventions, including volume administration, which is well recognized as lowering serum creatinine concentration particularly in patients with baseline impairment of kidney function; discontinuation of RAS blockade, increasing glomerular filtration due to differential effects on afferent and efferent arteriolar tone; and use of pharmacologic agents such as $\mathrm{N}$-acetyl cysteine [12] and statins [13], which may independently lower measured creatinine concentration through interference in laboratory assay, alteration tubular secretion, reduction in creatinine generation, or other mechanisms.

A variety of strategies may be used to overcome these shortcomings associated with small, short-term change in serum creatinine as an endpoint in AKI studies. The use of larger increments in serum creatinine obviates some of these issues. However, while reliance on larger changes in serum creatinine increases endpoint specificity, it is at the expense of a substantial decrease in event rates and a corresponding increase in the sample size 
required for an adequately powered clinical trial. Alternatively, combination of adequately validated biomarkers of kidney injury with small, short-term increases in serum creatinine could provide enhanced endpoint specificity through improved AKI phenotyping.

\section{Major Adverse Kidney Events}

The relationship between AKI and clinically important longer term outcomes, including death, need for dialysis, and persistent worsening of kidney function, serves as the basis for the use of these outcomes as endpoints for AKI prevention or treatment trials (Fig. 1b). These major adverse kidney events (MAKE), assessed at $30,60,90$, or even 180 days have been utilized as primary endpoints in several recent clinical trials [14-16]. However, some caution must be employed when using the MAKE endpoint. Ideally, components of a composite endpoint are of similar clinical importance. The MAKE endpoint combines mortality with both clinical intervention (need for dialysis) and biochemical change (increase in serum creatinine), outcomes of disparate significance. In addition, many factors in patients at risk for AKI, particularly in the critical care setting, may predispose to death. Thus, mortality as an endpoint may be insensitive to potential nephroprotective interventions. Despite these limitations, there is rationale for the inclusion of mortality, as it is a competing outcome for the other components. One strategy to address these concerns would be to treat the components in a hierarchal fashion. There are also limitations to inclusion of need for RRT as an endpoint, as criteria for initiation, continuation, and discontinuation of RRT are highly variable.

A second potential concern relates to ascertainment bias if change in kidney function is based on laboratory studies obtained as part of routine care rather than systematic measurement. For example, in 2 recent studies that utilized the MAKE endpoint assessed at 30 days, change in kidney function was assessed using the last inpatient value within 30 days of admission $[15,16]$. This may have resulted in over-estimation of event rates if patients were discharged prior to full recovery of kidney function. Reliance on outpatient laboratory values obtained as part of routine care may introduce bias, as patients at greater risk for kidney disease are likely to have more frequent creatinine testing.

Finally, while there is a strong association between AKI and subsequent development of its components, evidence demonstrating that this association is causal and that prevention of AKI decreases subsequent worsening of kidney function is scant [17]. Many risk factors for $\mathrm{AKI}$, including pre-existent $\mathrm{CKD}$, diabetes mellitus, and concomitant cardiovascular disease, are also independent risk factors for these outcomes. Intervening events during the follow-up period may also confound the relationship between an intervention and this endpoint, particularly as the interval between intervention and endpoint ascertainment increases (Fig. 1c). The validity of the MAKE endpoint as a primary outcome in trials may be strengthened, in some settings, if combined with ascertainment of AKI as a secondary outcome.

\section{Conclusion}

Unfortunately, there is no perfect endpoint for use in clinical trials for prevention or treatment of AKI. As highlighted above, there are significant limitations associated with both small, short-term changes in serum creatinine and with more definitive, longer term outcomes as endpoints for clinical trials of AKI. These issues need to be carefully considered when selecting endpoints in the design of trials, recognizing that the endpoint used in any specific trial must be selected with consideration of multiple factors, including both the population and intervention to be studied and the specific goals of the trial.

\section{Disclosure Statement}

Dr. Palevsky reports consulting for GE Healthcare, Baxter, and Novartis.

\section{References}

1 Bellomo R, Ronco C, Kellum JA, Mehta RL, Palevsky P; Acute Dialysis Quality Initiative Workgroup: Acute renal failure - definition, outcome measures, animal models, fluid therapy and information technology needs: the Second International Consensus Conference of the Acute Dialysis Quality Initiative (ADQI) Group. Crit Care 2004;8:R204R212.

2 Mehta RL, Kellum JA, Shah SV, Molitoris BA, Ronco C, Warnock DG, et al: Acute kidney injury network (AKIN): report of an initiative to improve outcomes in acute kidney injury. Crit Care 2007;11:R31.

3 Kidney Disease: Improving global outcomes (KDIGO) acute kidney injury work group. KDIGO clinical practice guideline for acute kidney injury. Kidney Int Suppl 2012;2:1138. 
4 Kellum JA, Zarbock A, Nadim MK: What endpoints should be used for clinical studies in acute kidney injury? Intensive Care Med 2017;43:901-903.

5 Mehta RL, Chertow GM: Acute renal failure definitions and classification: time for change? J Am Soc Nephrol 2003;14:21782187.

6 Coca SG, Garg AX, Swaminathan M, Garwood S, Hong K, Thiessen-Philbrook H, et al: Preoperative angiotensin-converting enzyme inhibitors and angiotensin receptor blocker use and acute kidney injury in patients undergoing cardiac surgery. Nephrol Dial Transplant 2013;28:2787-2799.

7 Testani JM, Kimmel SE, Dries DL, Coca SG: Prognostic importance of early worsening renal function after initiation of angiotensinconverting enzyme inhibitor therapy in patients with cardiac dysfunction. Circ Heart Fail 2011;4:685-691.

8 Al-Aly Z, Balasubramanian S, McDonald JR, Scherrer JF, O’Hare AM: Greater variability in kidney function is associated with an increased risk of death. Kidney Int 2012;82: 1208-1214.

9 Lacher DA, Hughes JP, Carroll MD: Biological variation of laboratory analytes based on the 1999-2002 National Health and Nutrition Examination Survey. Natl Health Stat Report 2010:1-7.

10 Lin J, Fernandez H, Shashaty MG, Negoianu D, Testani JM, Berns JS, et al: False-positive rate of AKI using consensus creatinine-based criteria. Clin J Am Soc Nephrol 2015;10: 1723-1731.

11 Marenzi G, Marana I, Lauri G, Assanelli E, Grazi M, Campodonico J, et al: The prevention of radiocontrast-agent-induced nephropathy by hemofiltration. N Engl J Med 2003;349:1333-1340.

12 Gonzales DA, Norsworthy KJ, Kern SJ, Banks S, Sieving PC, Star RA, et al: A meta-analysis of $\mathrm{N}$-acetylcysteine in contrast-induced nephrotoxicity: unsupervised clustering to resolve heterogeneity. BMC Med2007;5:32.
13 Vidt DG, Harris S, McTaggart F, Ditmarsch M, Sager PT, Sorof JM: Effect of short-term rosuvastatin treatment on estimated glomerular filtration rate. Am J Cardiol 2006;97: 1602-1606.

14 Weisbord SD, Gallagher M, Jneid H, Garcia S, Cass A, Thwin SS, et al: Outcomes after angiography with sodium bicarbonate and acetylcysteine. N Engl J Med 2018;378:603-614.

15 Self WH, Semler MW, Wanderer JP, Wang L, Byrne DW, Collins SP, et al: Balanced crystalloids versus saline in noncritically Ill adults. N Engl J Med 2018;378:819-828.

16 Semler MW, Self WH, Wanderer JP, Ehrenfeld JM, Wang L, Byrne DW, et al: Balanced crystalloids versus saline in critically Ill adults. N Engl J Med 2018;378:829-839.

17 Coca SG, Zabetian A, Ferket BS, Zhou J, Tes$\operatorname{tani}$ JM, Garg AX, et al: Evaluation of shortterm changes in serum creatinine level as a meaningful end point in randomized clinical trials. J Am Soc Nephrol 2016;27:25292542 . 